\title{
Multiple Marker Effects of Single Nucleotide Polymorphisms in Two Genes, NCAPG and PLAG1, for Carcass Weight in Japanese Black Cattle
}

\author{
Youji Muramatsu \\ Department of Nutritional Sciences for Well-being, Faculty of Health Sciences for Welfare, Kansai University of Welfare Sciences, \\ Kashiwara, Osaka, Japan \\ Email: *muramatsu@tamateyama.ac.jp
}

How to cite this paper: Muramatsu, Y. (2019) Multiple Marker Effects of Single Nucleotide Polymorphisms in Two Genes, NCAPG and PLAG1, for Carcass Weight in Japanese Black Cattle. Open Journal of Animal Sciences, 9, 129-134.

https://doi.org/10.4236/ojas.2019.91011

Received: December 15, 2018

Accepted: January 12, 2019

Published: January 15, 2019

Copyright $\odot 2019$ by author(s) and Scientific Research Publishing Inc. This work is licensed under the Creative Commons Attribution International License (CC BY 4.0).

http://creativecommons.org/licenses/by/4.0/

\begin{abstract}
Carcass weight is an economically important trait for beef cattle breeding and markets. The previous studies detected two single nucleotide polymorphisms (SNPs) associated with carcass weight of Japanese Black: c.1326T> $G$ in NCAPG gene and FJX_250879 in PLAG1 gene. Here, I carried out multiple marker association analysis for the two SNPs in Japanese Black population of 218 animals. The multiple marker analysis with the model including the main effects of the two SNPs and their interaction detected significant main effects of $c .1326 T>G$ and $F J X \_250879$ and a significant interaction between the two SNPs, for carcass weight. These findings suggest the presence of inter-allelic interactions among genes affecting the variation of carcass weight. For effective marker-assisted selection for beef production, interaction between the two markers needs to be considered.
\end{abstract}

\section{Keywords}

Association, Beef Cattle, Carcass Weight, Interaction, SNP

\section{Introduction}

Carcass weight is one of economically important traits for beef cattle [1]. The detection of genes affecting carcass weight and the establishment of an effective marker-assisted selection technique based on the genomic information are an important goal for the genetic improvement of beef production.

Setoguchi et al. [2] identified the $c .1326 T>G$ single nucleotide polymorphism (SNP) in the non-SMC condensin I complex, subunit G (NCAPG) gene, which 
changes the amino acid Ile442 to Met442 in the encoded protein, as a candidate causative variation for a bovine carcass weight quantitative trait locus (QTL) on chromosome 6. In addition, Karim et al. [3] identified the stature quantitative trait nucleotide $(\mathrm{QTN})$ in the pleiomorphic adenoma gene 1 (PLAGI)-coiledcoil-helix-coiled-coil-helix domain containing 7 ( $C H C H D 7)$ intergenic region, as the causative variation for another carcass weight QTL on chromosome 14. Recently, Hoshiba et al. [4] compared the effects on growth-related traits of the c.1326T>G SNP and the $F J X \_250879$ SNP that was approximately 50 -kb centromeric from the stature QTN and nearly linkage disequilibrium with the QTN on chromosome 14, using Japanese Black steers. As a result, they detected a genetic interaction between the $c .1326 T>G$ and $F J X \_250879$ SNPs for body and rump lengths.

Because the two SNPs have relatively large effects on carcass weight, it is important to examine whether a genetic interaction for carcass weight is present between them. Thus, I performed multiple marker analysis for association with carcass weight, using the model including the main effects of the two SNPs and their interaction in Japanese Black beef cattle.

\section{Materials and Methods}

\subsection{Samples and Data}

Two hundred eighteen paternal half-sib Japanese Black steers were produced from 53 sires ( 1 - 16 steers per sire) in Niigata prefecture. Hair root specimens of the progeny steers were provided from Niigata Agricultural Research Institute Livestock Research Center, and were used for genotyping the SNPs. DNA samples were prepared from the materials using DNeasy Blood \& Tissue kit (Qiagen, Hilden, Germany), according to the manufacturer's instructions. The carcass weight was measured according to the Japanese meat grading system by certified graders from the Japan Meat Grading Association (Tokyo, Japan) [1]. The records of carcass weight for the progeny steers were obtained from the Niigata Prefectural Headquarters, National Federation of Agricultural Cooperative Association (Niigata, Japan).

\subsection{SNP Genotyping}

The $c .1326 T>G$ SNP was genotyped by the PCR-restriction fragment length polymorphism method as described previously [5]. The FJX_250879 SNP was genotyped by direct sequencing of the PCR products according to Nishimura et al. [6]. Primers used in this study were shown in Table 1. In the Japanese Black population, the $C$-allele of $F J X \_250879$ increases carcass weight and corresponds to the $Q$ (superior) allele of the stature QTN [6].

\subsection{Association Analysis}

I performed association analysis with multiple marker model of the two SNPs, c.1326T>G and FJX_250879, in which carcass weight records were used as the 
Table 1. PCR primers used in this study.

\begin{tabular}{cc}
\hline SNP & Sequences \\
\hline c.1326T>G & 5'-ATT TAG GAA ACG ACT ACT GG-3' \\
5'-ATT TGT ATT CTC TTA TTA TCA TC-3' \\
FJ__250879 \\
5'-ATG GGA TCA CCA CAG ACC AT-3' \\
5'-TGC ACA GAA TCA GTG TGT CTT TT-3'
\end{tabular}

dependent variable. The model included the two SNPs and their gene to gene (inter-allelic) interaction effect between the SNPs, slaughter years and months, and fattening farms as the fixed effects, and the sires as the random effect. The slaughter age of the animals was included as a covariate in linear regression. The analysis was performed by the MIXED procedure of the SAS program (SAS Institute, Inc., Cary, NC).

\section{Results and Discussion}

Using the multiple marker association analysis, I analyzed the main effects of the two SNPs, c.1326T>G and FJX_250879, and their gene to gene interaction effect for carcass weight. The main effects of $c .1326 T>G$ and $F J X \_250879$ were statistically significant ( $P=0.0272$ for $c .1326 T>G$ and $P=0.0235$ for $F J X \_250879$ ) (Table 2). The least-squares mean of the $G G$ homozygote of $c .1326 T>G$ was significantly higher than the mean of the TT homozygote, and the mean of the heterozygote was intermediate. The mean of the CC homozygote of FJX_250879 was significantly higher than that of the $G G$, and the mean of the heterozygote was intermediate. These results correspond with the previous studies [2] [6] where it was concluded that the favorable alleles for carcass weight were $G$ at $c .1326 T>G$ and $C$ at $F J X \_250879$. The interaction between $c .1326 T>G$ and $F J X \_250879$ was statistically significant for carcass weight $(P=0.0147)$ (Table 2$)$. The combination of $T T$ at $c .1326 T>G$ and $G G$ at $F J X \_250879$ gave a marked decrease.

In the multiple marker analysis of this study, $c .1326 T>G$ and $F J X \_250879$ had significant main effects on carcass weight with the same favorable tendency of alleles as previous studies [2] [6]. Further, I detected a significant epistatic interaction between $c .1326 T>G$ and $F J X \_250879$ for carcass weight. These findings suggest that $c .1326 T>G$ and $F J X \_250879$, respectively, affect carcass weight both directly and through its interaction with $F J X \_250879$ and $c .1326 T>G$.

The maximum difference between the largest and smallest combined genotypic effects of the two SNPs was expected as 101.96 in carcass weight scale (= $33.10-[-68.86]$; see below) based on Table 2. The largest effect came from the combination of $G G$ at $c .1326 T>G(5.40)$ and $C C$ at $F J X \_250879$ (10.72), and the interaction between $G G$ at $c .1326 T>G$ and $C C$ at $F J X \_250879$ (16.98) (i.e. $5.40+$ $10.72+16.98=33.10)$. The smallest effect came from the respective combination of $T T(-7.45)$ and $G G(-14.78)$, and the interaction between $T T$ at $c .1326 T>G$ and $G G$ at $F J X \_250879(-46.63)($ i.e. $[-7.45]+[-14.78]+[-46.63]=[-68.86])$. 
Table 2. Main and interaction effects of the two single nucleotide polymorphisms (SNPs) for carcass weight in the multiple marker analysis using 218 animals.

\begin{tabular}{|c|c|c|c|}
\hline SNP & Genotype & $n^{1}$ & $\mathrm{LSM}^{2}$ \\
\hline c. $1326 T>G$ & $T T$ & 102 & $-7.45 \pm 4.13^{\mathrm{a}}$ \\
\hline \multirow[t]{2}{*}{$(P=0.0272)$} & $T G$ & 98 & $2.05 \pm 2.91^{\mathrm{b}}$ \\
\hline & $G G$ & 18 & $5.40 \pm 2.12^{c}$ \\
\hline$F J X \_250879$ & $G G$ & 63 & $-14.78 \pm 3.75^{\mathrm{a}}$ \\
\hline \multirow[t]{2}{*}{$(P=0.0235)$} & $G C$ & 106 & $4.07 \pm 3.07^{\mathrm{b}}$ \\
\hline & $C C$ & 49 & $10.72 \pm 4.21^{\mathrm{c}}$ \\
\hline c.1326T>G\& FJX_250879 & $T T \& G G$ & 26 & $-46.63 \pm 30.70^{a}$ \\
\hline \multirow[t]{8}{*}{$(P=0.0147)$} & $T T \& G C$ & 51 & $12.52 \pm 7.68^{\mathrm{b}}$ \\
\hline & $T T \& C C$ & 25 & $19.54 \pm 17.73^{\mathrm{b}}$ \\
\hline & $T G \& G G$ & 32 & $-8.13 \pm 15.70^{\mathrm{ab}}$ \\
\hline & $T G \& G C$ & 46 & $12.65 \pm 7.04^{\mathrm{b}}$ \\
\hline & $T G \& C C$ & 20 & $18.21 \pm 12.34^{\mathrm{b}}$ \\
\hline & $G G \& G G$ & 5 & $27.77 \pm 15.35^{\mathrm{b}}$ \\
\hline & $G G \& G C$ & 9 & $11.17 \pm 8.21^{\mathrm{b}}$ \\
\hline & $G G \& C C$ & 4 & $16.98 \pm 11.56^{\mathrm{b}}$ \\
\hline
\end{tabular}

${ }^{1}$ Number of genotyped animals. ${ }^{2}$ Least-squares mean (LSM) \pm SE of carcass weight. LSMs are shown as the deviation from the general mean of carcass weight. ${ }^{\text {ab, }, c}$ Statistically significant among means with different superscripts $(P<0.05)$. $p$ value was shown in the parentheses.

An epistatic interaction is measured as the departure of the combined effects of two or more genes from the sum of their individual effects [7]. This phenomenon is thought to play a significant role in evolution [8] [9]. Matsuhashi et al. [10] recently clarified multiple marker effects of four gene polymorphisms on the fatty acid composition and several carcass traits in Japanese Black cattle, but detected no epistatic interactions. However, I detected a possible epistatic interaction for carcass weight between $N C A P G($ c.1326T>G) and PLAG1 (FJX_250879), the strongest candidate gene for the carcass weight QTL on chromosome 14. Although the functional relevance of such an interaction is still unknown, NCAPG is involved in arginine metabolism [11] and PLAG1 in regulation of the expression of growth factors, including insulin-like growth factor 2 [12]. An interaction between them could play an important role in the variation of carcass weight in beef cattle. For confirmation of the presence of the interaction, other replication studies are recommended using other Japanese Black populations. In addition, a further investigation on detection of an epistatic interaction among three or more genes affecting the variation of carcass weight would be required for effective marker-assisted selection.

\section{Conclusion}

In conclusion, I have demonstrated significant associations and interaction of 
two SNPs, referred to as $c .1326 T>G$ and $F J X \_250879$, for carcass weight in Japanese Black population. For the effective genetic improvement of beef production in Wagyu population, the role of interactions among SNP markers under marker-assisted selection should be considered.

\section{Acknowledgements}

I would like to give my sincere thanks to Prof. Takahisa Yamada of Niigata University for his valuable guidance of this work.

\section{Conflicts of Interest}

The authors declare no conflicts of interest regarding the publication of this paper.

\section{References}

[1] JMGA (1988) New Beef Carcass Grading Standards. Japan Meat Grading Association, Tokyo.

[2] Setoguchi, K., Furuta, M., Hirano, T., Nagao, T., Watanabe, T., Sugimoto, Y. and Takasuga, A. (2009) Cross-Breed Comparisons Identified a Critical 591-kb Region for Bovine Carcass Weight QTL (CW-2) on Chromosome 6 and the Ile-442-Met Substitution in NCAPG as a Positional Candidate. BMC Genetics, 10, 43. https://doi.org/10.1186/1471-2156-10-43

[3] Karim, L., Takeda, H., Lin, L., Druet, T., Arias, J.A., Baurain, D., Cambisano, N., Davis, S.R., Farnir, F., Grisart, B., Harris, B.L., Keehan, M.D., Littlejohn, M.D., Spelman, R.J., Georges, M. and Coppieters, W. (2011) Variants Modulating the Expression of a Chromosome Domain Encompassing PLAG1 Influence Bovine Stature. Nature Genetics, 43, 405-413. https://doi.org/10.1038/ng.814

[4] Hoshiba, H., Setoguchi, K., Watanabe, T., Kinoshita, A., Mizoshita, K., Sugimoto, Y. and Takasuga, A. (2013) Comparison of the effects explained by variations in the bovine PLAG1 and NCAPG genes on daily body weight gain, linear skeletal measurements and carcass traits in Japanese Black steers from a progeny testing program. Animal Science Journal, 84, 529-534. https://doi.org/10.1111/asj.12033

[5] Setoguchi, K., Watanabe, T., Weikard, R., Albrecht, E., Kühn, C., Kinoshita, A., Sugimoto, Y. and Takasuga, A. (2011) The SNP c.1326T> G in the Non-SMC Condensin I Complex, Subunit G (NCAPG) Gene Encoding a p.Ile442Met Variant Is Associated with an Increase in Body Frame Size at Puberty in Cattle. Animal Genetics, 42, 650-655. https://doi.org/10.1111/j.1365-2052.2011.02196.x

[6] Nishimura, S., Watanabe, T., Mizoshita, K., Tatsuda, K., Fujita, T., Watanabe, N., Sugimoto, Y. and Takasuga, A. (2012) Genome-Wide Association Study Identified Three Major QTL for Carcass Weight Including the PLAG1-CHCHD7 QTN for Stature in Japanese Black Cattle. BMC Genetics, 13, 40. https://doi.org/10.1186/1471-2156-13-40

[7] Frankel, W.N. and Schork, N.J. (1996) Who's Afraid of Epistasis? Nature Genetics, 14, 371-373. https://doi.org/10.1038/ng1296-371

[8] Rieseberg, L.H., Sinervo, B., Linder, C.R., Ungerer, M.C. and Arias, D.M. (1996) Role of Gene Interactions in Hybrid Speciation: Evidence from Ancient and Experimental Hybrids. Science, 272, 741-745.

https://doi.org/10.1126/science.272.5262.741 
[9] Schork, N.J. (1997) Genetically Complex Cardiovascular Traits. Origins, Problems, and Potential Solutions. Hypertension, 29, 145-149.

https://doi.org/10.1161/01.HYP.29.1.145

[10] Matsuhashi, T., Maruyama, S., Uemoto, Y., Kobayashi, N., Mannen, H., Abe, T., Sakaguchi, S. and Kobayashi, E. (2011) Effects of Bovine Fatty Acid Synthase, Stearoyl-Coenzyme A Desaturase, Sterol Regulatory Element-Binding Protein 1, and Growth Hormone Gene Polymorphisms on Fatty Acid Composition and Carcass Traits in Japanese Black Cattle. Journal of Animal Science, 89, 12-22. https://doi.org/10.2527/jas.2010-3121

[11] Weikard, R., Altmaier, E., Suhre, K., Weinberger, K.M., Hammon, H.M., Albrecht, E., Setoguchi, K., Takasuga, A. and Kühn, C. (2010) Metabolomic Profiles Indicate Distinct Physiological Pathways Affected by Two Loci with Major Divergent Effect on Bos taurus Growth and Lipid Deposition. Physiological Genomics, 42, 79-88. https://doi.org/10.1152/physiolgenomics.00120.2010

[12] Voz, M.L., Mathys, J., Hensen, K., Pendeville, H., Van Valckenborgh, I., Van Huffel, C., Chavez, M., Van Damme, B., De Moor, B., Moreau, Y. and Van de Ven, W.J. (2004) Microarray Screening for Target Genes of the Proto-Oncogene PLAG1. Oncogene, 23, 179-191. https://doi.org/10.1038/sj.onc.1207013 